\title{
Bed regime as a lifesaving factor in spontaneous intracranial hypotension
}

\author{
Grzegorz Turek ${ }^{1}$, Adrian Rogala ${ }^{1}$, Mateusz Ząbek ${ }^{2}$, Mirosław Ząbek ${ }^{1,3,4}$ \\ ${ }^{1}$ Department of Neurosurgery, Brodno Masovian Hospital, Warsaw, Poland \\ ${ }^{2}$ Faculty of Medicine, Collegium Medicum, Cardinal Stefan Wyszynski University, Warsaw, Poland \\ ${ }^{3}$ Gamma Knife Centre, Warsaw, Poland \\ ${ }^{4}$ Department of Neurosurgery, Postgraduate Medical Centre, Warsaw, Poland
}

Key words: $\mathrm{SIH}$, headache, dural tear, bed rest

(Neurol Neurochir Pol 2021; 55 (4): 407-409)

\section{To the Editors}

Spontaneous intracranial hypotension $(\mathrm{SIH})$ was first described by Schalltenbrand in 1938. It is a clinical disorder in which cerebrospinal fluid (CSF) volume or pressure drop down due to a dural CSF leakage [1]. Although historically considered a rare condition, SIH has been increasingly recognised, with an incidence estimated at 5 per 100,000 [2]. However, it is frequently initially misdiagnosed [3]. A large number of symptoms can lead to difficulties in diagnosis. It is extremely important to pay particular attention to the fact that symptoms may mimic acute cerebrovascular events (ACE). The condition is characterised by similar symptoms. Research shows an incorrect diagnosis in $66.3 \%$. Incorrect diagnoses are mainly made in the pre-hospital setting, but can also happen in the ED, especially if the treating physician is not a neurologist or stroke physician [4]. The clinical hallmark is debilitating postural headache, exacerbated by standing, and relieved in the recumbent position. Patients may also present with diplopia, tinnitus, vertigo, dizziness, neck stiffness, nausea, vomiting, hyperacusis, deafness, and vision loss [5]. Conservative treatment is limited to bed rest, hydration, caffeine and analgesic drugs [6]. In this letter, we present life-threatening complications of SIH in two patients.

\section{Case 1}

A 42-year-old female was admitted to hospital with a two-week history of headache that was persistent, changed depending on the patient's position, and was posture related. The patient also reported nausea and vomiting. No abnormalities were found in blood test or neurological examination, nor did the patient have a history of head or neck trauma. Computed tomography of the brain (CT) revealed bilateral hypodense subdural collections of blood ( $4 \mathrm{~mm}$ and $5 \mathrm{~mm}$ ), along with the obliteration of the prepontine cistern. Initially, the patient was prescribed bed rest, hydration and steroid therapy. Unfortunately, she did not comply with these. Brain MRI demonstrated an advanced descent of the brainstem with flattening of the pontine surface, dilation of the sagittal sinus, enlargement of the pituitary gland, and expansion of the subdural haematomas (Fig. 1A). Additionally, C6/C7 disc herniation was found on spinal MRI, without accompanying symptoms. Isotopic cisternography and CT myelography results were normal. However, the patient again failed to comply with a strict bed regime. As a result, her score on the Glasgow Coma Scale (GCS) decreased from 15 to 14 . CT confirmed the IH and enlargement of the subdural haematomas $(7 \mathrm{~mm}$ and $11 \mathrm{~mm})$. The patient still did not follow medical orders and a decrease to GCS 7 was recorded. As the aetiology of SIH remained unknown, the

Address for correspondence: Adrian Rogala, Department of Neurosurgery, Brodno Masovian Hospital, Ludwika Kondratowicza 8, 03-242 Warsaw, Poland; e-mail: adrian.kamil.rogala@gmail.com

Received: 27.03.2021 Accepted: 17.05.2021 Early publication date: 10.06.2021 
patient was implanted with a both-sided subdural-peritoneal shunt, which caused a transient increase in GCS score up to 15. But the patient's neurological condition again deteriorated to GCS 5 four days after the implantation. This was primarily a consequence of further non-compliance with the bed regime. When emergency decompressive craniectomy was performed, the patient improved to 15 GCS. Another MRI with T2-weighted sequences showed the cause of SIH, a collection of cerebrospinal fluid referred as a double dural sac sign' in the extradural space, just above the degenerated C6/C7 segment (Fig. 1B). However, no epidural blood patch (EBP) was carried out at the affected segment of the spine, given the risk of neurological complications. The decision not to perform EBP was driven by the review paper published by Kapoor et al. [7]. Instead, anterior cervical discectomy and fusion at $\mathrm{C} 6 / \mathrm{C} 7$, along with the inspection of dura mater at that level, were performed.

A large collection of cerebrospinal fluid was found extradurally, but no evident tear of the dura was identified despite a thorough inspection of the dural sac at the level of the disc herniation. The dura watertight protection was obtained by sealant matrix TachoSil. Despite further non-compliance with the bed regime, no deterioration of the patient's neurological condition was observed post-surgery. The patient was discharged with a GCS score of 15 . No signs of SIH were found on a follow-up MRI six months later.

\section{Case 2}

A 54-year-old male presented with a four-week history of postural headache, concomitant nausea and vomiting, without neurological signs and symptoms, head or neck trauma. The results of blood tests, levels of paraneoplastic markers, thoracic radiogram, and abdominal ultrasound findings were normal. An initial CT of the brain revealed bilateral hypodense subdural collections of blood $(8 \mathrm{~mm}$ each side) and the prepontine cistern's obliteration. Initially, the patient was treated with hydration, steroid therapy and ordered a strict bed regime. Failure to comply with medical recommendations contributed to the suspicion of IH. MRI revealed a critical descent of the brainstem with flattening of the pontine surface and enlargement of the pituitary gland. After the administration of a gadolinium-based contrast agent, diffuse pachymeningeal enhancement in the supratentorial and infratentorial regions was observed, ultimately confirming the diagnosis of IH. Spinal MRI showed advanced degenerative disease of the cervical spine. Subsequent CT myelography revealed a significant CSF leakage at the level of $\mathrm{C} 1 / \mathrm{C} 2$ (Fig. 1C). To reduce the risk of a spinal cord injury, the patient was qualified to laminectomy with inspection of the dura at the leakage level, rather than to EBP. At the time of the qualification, the patient's GCS score was 15 . However, the patient did not comply with the bed regime and his condition suddenly deteriorated, GCS score dropping from

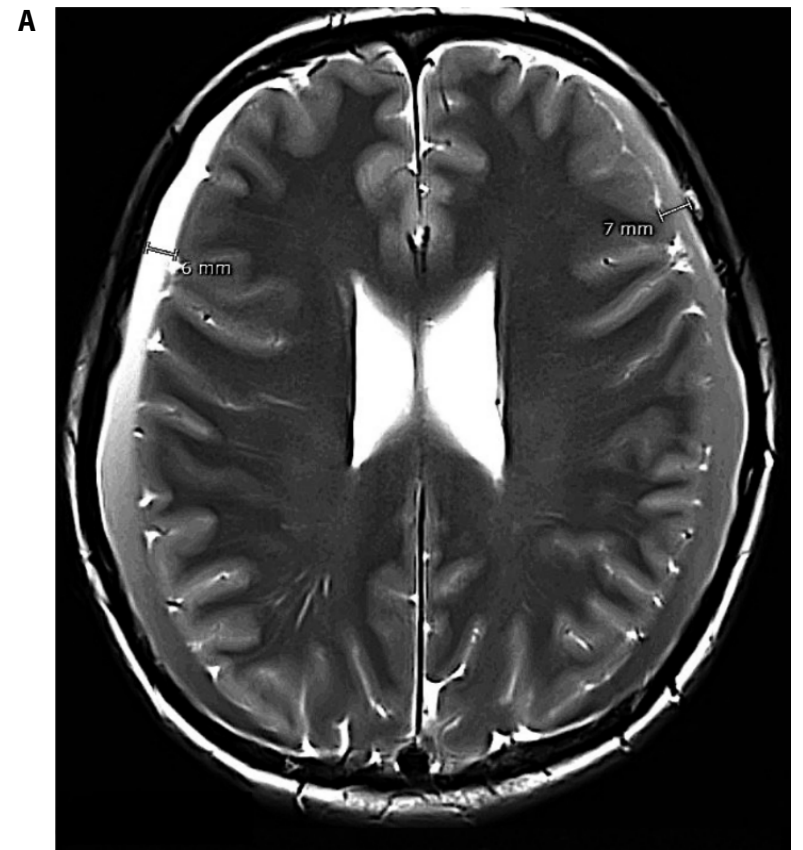

B

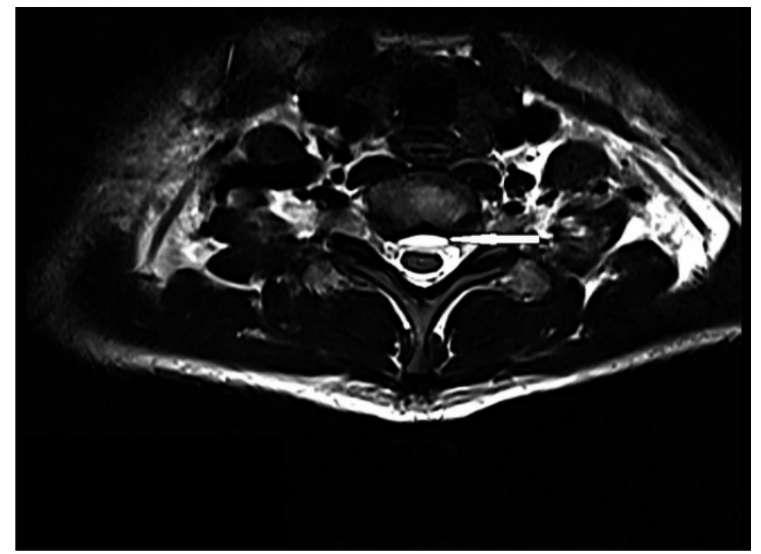

C

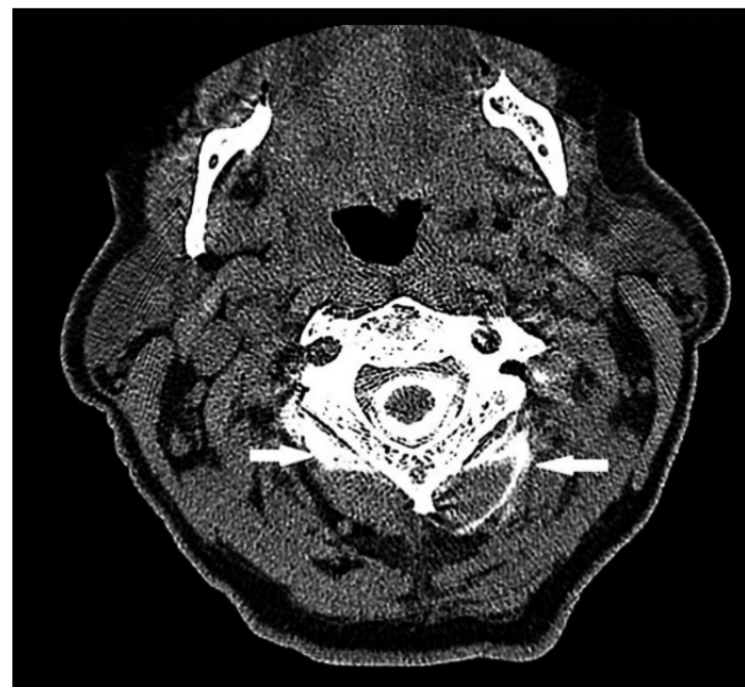

Figure 1. A. MRI showing expansion of subdural haematoma - case 1; B. MRI with T2-weighted sequences showed collection of CSF within extradural space, just above degenerated C6/C7 segment ('double dural sac sign') - case 1; C. CT myelography revealed significant CSF leakage at level of $\mathrm{C} 1 / \mathrm{C} 2$ - case 2 
15 to 7 within an hour. Control CT revealed a critical descent of cerebellar tonsils to the foramen magnum; the patient was already unconscious at that time, with a GCS score of 7. Large emergency decompressive craniectomy of the posterior fossa with the removal of the posterior edge of the foramen magnum was performed. During subsequent C1-C4 laminectomy, CSF leakage from a large dural laceration at the $\mathrm{C} 2$ nerve root level was identified. The laceration was thoroughly sealed by collagen matrix coated with human fibrinogen and thrombin - TachoSil. The subdural collections of blood were evacuated through both-sided decompressive craniectomy. The patient was transferred to the intensive care unit and put into a propofol-induced coma for five days. After recovery from the coma, he was fully conscious, with a GCS score of 15 .

A rare condition, such as $\mathrm{IH}$, can constitute a diagnostic and therapeutic challenge. IH is highly likely to be misdiagnosed or overlooked because patients with this condition usually lack objective symptoms. No clear guidelines have been published with regard to the conservative management of $\mathrm{IH}$. While according to some authors an approximately two-week bed rest is sufficient to control this condition $[8,9]$, some published evidence suggests that conservative treatment might not be enough [10]. The most likely reason behind these discrepancies is patient non-compliance with the bed regime. This is well illustrated by the two cases presented herein; lack of compliance with the bed regime resulted in further CSF leakage, brain sagging and deterioration of the clinical condition, as shown by a rapid decrease in GCS scores. To the best of our knowledge, non-compliance with physician's orders has not been reported as a cause of IH exacerbation to date, and this case series is the first published evidence of potentially fatal complications related to non-compliance with a strict bed regime. While CSF leakage is a major cause of SIH, the underlying mechanisms are yet to be elucidated. Supine position of the body creates favourable conditions for dura mater regeneration, improving the CSF fluctuation and shifting the centre of mass of the brain upward $[11,12]$.

A bed regime seems to be an effective and vital component of IH treatment [13]. As we have presented in this paper, non-compliance with the bed regime can lead to life-threatening complications.

\section{References}

1. Schalltenbrand G. Neuere anschauungen zur pathophysiologie der liquozirkulation. Zentralbl Neurochir. 1938; 3: 290-300.

2. Kranz PG, Gray L, Amrhein TJ. Spontaneous intracranial hypotension: 10 myths and misperceptions. Headache. 2018; 58(7): 948-959, doi: 10.1111/head.13328, indexed in Pubmed: 29797515.

3. Schievink WI. Misdiagnosis of spontaneous intracranial hypotension. Arch Neurol. 2003; 60(12): 1713-1718, doi: 10.1001/archneur.60.12.1713, indexed in Pubmed: 14676045.

4. Kozera-Strzelińska D, Karliński M, Rak G, et al. Stroke and TIA mimics in patients referred to a neurological emergency department by non-ambulance physicians, ambulance physicians and paramedics. Neurol Neurochir Pol. 2019; 53(1): 83-89, doi: 10.5603/PJNNS. a2019.0002, indexed in Pubmed: 30614515.

5. Haritanti A, Karacostas D, Drevelengas A, et al. Spontaneous intracranial hypotension: clinical and neuroimaging findings in six cases with literature review. Eur J Radiol. 2009; 69(2): 253-259, doi: 10.1016/j.ejrad.2007.10.013, indexed in Pubmed: 18182266.

6. Williams EC, Buchbinder BR, Ahmed S, et al. Spontaneous intracranial hypotension: presentation, diagnosis, and treatment. Anesthesiology. 2014; 121(6): 1327-1333, doi: 10.1097/ ALN.0000000000000410, indexed in Pubmed: 25118954.

7. Kapoor SG, Ahmed S. Cervical epidural blood patch - a literature review. Pain Med. 2015; 16(10): 1897-1904, doi: 10.1111/ pme.12793, indexed in Pubmed: 26122010.

8. Liang H, Xu C, Liu T, etal. Spontaneous intracranial hypotension in Hashimoto's thyroiditis: a case report. Medicine (Baltimore). 2019; 98(18): e15476, doi: 10.1097/MD.0000000000015476, indexed in Pubmed: 31045829.

9. Sugino T, Matsusaka Y, Mitsuhashi Y, et al. Intracranial hypotension due to cerebrospinal fluid leakage detected by radioisotope cisternography. Neurol Med Chir (Tokyo). 2000; 40(8): 404-407, doi: 10.2176/nmc.40.404, indexed in Pubmed: 10979262.

10. Zheng Y, Lian Y, Wu C, et al. Diagnosis and treatment of spontaneous intracranial hypotension due to cerebrospinal fluid leakage. SpringerpIus. 2016; 5(1): 2108, doi: 10.1186/s40064-016-3775-z, indexed in Pubmed: 28066697.

11. Whedon JM, Glassey D. Cerebrospinal fluid stasis and its clinical significance. Altern Ther Health Med. 2009; 15(3): 54-60, indexed in Pubmed: 19472865.

12. Roberts DR, Zhu X, Tabesh A, et al. Structural brain changes following long-term $6^{\circ}$ head-down tilt bed rest as an analog for spaceflight. AJNR Am J Neuroradiol. 2015; 36(11): 2048-2054, doi: 10.3174/ ajnr.A4406, indexed in Pubmed: 26185326.

13. Hoffmann J, Goadsby PJ. Update on intracranial hypertension and hypotension. Curr Opin Neurol. 2013; 26(3): 240-247, doi: 10.1097/ WCO.0b013e328360eccc, indexed in Pubmed: 23594732. 